\title{
A Dynamic Model of a Reinforced Thin Plate with Ribs of Finite Width
}

\author{
Jaclyn E. Sylvia and Andrew J. Hull \\ Naval Undersea Warfare Center, Newport, Rhode Island, USA
}

(Received 1 March 2012; Revised 27 February 2013; Accepted 19 March 2013)

\begin{abstract}
This paper derives an analytical model of a thin plate of infinite extent stiffened by ribs that have a finite width. The governing model of the plate is the one-dimensional flexural wave equation, and the ribs were modelled as translational springs. The plate was excited by an external load that is harmonic in time and space. An orthogonalization procedure was developed, and the resulting system equations are an infinite set of algebraic equations containing a diagonal matrix that represents the plate dynamics and a full matrix that contains permutations of the Fourier coefficients of the Heaviside step function that represent the rib forces. This matrix equation is truncated and inverted and yields a solution of the plate displacements. An example problem is formulated, and the resulting displacement field is compared to a solution generated using finite element analysis for validation of the solution method. The system stop- and pass-band behaviour as a function of rib width is illustrated. It is shown that an increase in the width of the ribs results in greater stop-band behaviour of the system.
\end{abstract}

\section{NOMENCLATURE}

$\begin{array}{ll}A_{p} & \text { Dynamic plate term } \\ b & \text { Width of each rib } \\ c_{n} & \text { Fourier coefficients of the Heaviside step function } \\ D & \text { Flexural rigidity of the plate } \\ E & \text { Young's modulus of the plate } \\ f(x, t) & \text { External load applied to the plate } \\ F_{0} & \text { Magnitude of applied force } \\ h & \text { Height of the plate } \\ H & \text { Heaviside step function } \\ i & \text { Square root of }-1 \\ k & \text { Wavenumber of excitation } \\ k_{n} & \text { Periodic wavenumber term } \\ K & \text { Stiffness of each rib per unit length } \\ L & \text { Distance between adjacent ribs } \\ m & \text { Index } \\ n & \text { Index } \\ p & \text { Index } \\ t & \text { Time } \\ w(x, t) & \text { Transverse displacement of the plate } \\ W(x) & \text { Spatial term of displacement of the plate } \\ W_{m} & \text { Magnitude of indexed spatial term of } \\ & \text { displacement of the plate } \\ x & \text { Spatial location on plate } \\ v & \text { Density of the plate } \\ & \text { Poisson's ratio of the plate }\end{array}$

\section{INTRODUCTION}

Reinforced plates and shells have a variety of applications. For instance, they are present in the design of ships, undersea vehicles, and aircrafts because reinforcement provides increased structural strength with minimal increased weight. While reinforcing a structure will allow it to survive in environments in which an unreinforced structure will fail, the resulting dynamic response of the structure changes dramatically.
Early work in the area of reinforced plates generally modelled the reinforcement of the plate as a line stiffener using a Dirac delta function to mathematically represent the effects. ${ }^{1-6}$ Mace modelled the response of periodically stiffened fluidloaded plates to harmonic loading and to line and point force loading. ${ }^{1,2}$ Mace's work involved a transformation into the wavenumber domain and the evaluation of a contour integral. Stepanishen modelled the scattering characteristics of a plate with line impedance discontinuities. ${ }^{3}$ To evaluate the scattered pressure, Stepanishen used a Fourier integral to calculate the relationship between the plate velocity in the wavenumber domain and the spatial domain. Eatwell and Butler determined the sound radiation from a fluid-loaded plate stiffened by a finite number of beams. ${ }^{4}$ Their solution involves an asymptotic evaluation of the pressure field by two Fourier integrals. Cray determined the response of a sectionally aperiodic plate to a line force in the wavenumber domain. ${ }^{5}$ Recently, Hull derived the elastic response of a thick plate system to harmonic loading with stiffeners. ${ }^{6}$ Some work exists in which the stiffeners are modelled with finite width. Woolley modelled the acoustical scattering from a plate reinforced by a single rib and by a finite number of ribs. ${ }^{7,8}$ In these studies, Woolley formulates the problem in the wavenumber domain and then solves it using a complicated method of contour integration. Woolley allows ribs of finite width in his model; however, Woolley states that Stepanishen "has obtained a different result" (p. $1657 \mathrm{in}^{8}$ ). The specific problem of a spatial domain response of a thin plate reinforced by ribs of finite width subjected to convective loading has not yet been addressed.

This paper derives an analytical model of a thin plate of infinite spatial extent stiffened by periodic ribs that have a finite width. The model developed here is different from previous models as it is based on differential equation theory in the spatial domain only and thus does not involve a complicated integral to be evaluated. The governing equation is a flexural plate model that has an external load and is reinforced by an infinite 